\title{
Nao-Xue-Shu Oral Liquid Protects and Improves Secondary Brain Insults of Hypertensive Cerebral Hemorrhage
}

\author{
Hongning Jiang, ${ }^{1}$ Ying Qin, ${ }^{2}$ Te Liu, ${ }^{3}$ Liang Zhang, ${ }^{4}$ Mingzhe Wang, ${ }^{5}$ Baofeng Qin, \\ Wenfei Jiang, ${ }^{5}$ Weilong Liao, ${ }^{5}$ and Weidong Pan ${ }^{5}$ \\ ${ }^{1}$ Department of Radiology, Shuguang Hospital, Shanghai Traditional Chinese Medicine, Shanghai 201203, China \\ ${ }^{2}$ Department of Respiratory Medicine, Pudong Hospital of Traditional Chinese Medicine, Shanghai 201209, China \\ ${ }^{3}$ Shanghai Geriatric Institute of Chinese Medicine, Longhua Hospital, Shanghai University of Traditional Chinese Medicine, \\ Shanghai 200031, China \\ ${ }^{4}$ Department of Neurology, Shanghai Seventh Hospital, Shanghai University of Traditional Chinese Medicine, Shanghai 200137, China \\ ${ }^{5}$ Department of Neurology, Shuguang Hospital, Shanghai Traditional Chinese Medicine, Shanghai 201203, China
}

Correspondence should be addressed to Weidong Pan; panwd@medmail.com.cn

Received 13 December 2015; Revised 19 February 2016; Accepted 10 March 2016

Academic Editor: Giuseppe Esposito

Copyright (c) 2016 Hongning Jiang et al. This is an open access article distributed under the Creative Commons Attribution License, which permits unrestricted use, distribution, and reproduction in any medium, provided the original work is properly cited.

Aim. To determine one traditional Chinese medicine (TCM) Nao-Xue-Shu oral liquid which protects and improves secondary brain insults (SBI) in hypertensive cerebral hemorrhage $(\mathrm{HCH})$. Methods. 158 patients with $\mathrm{HCH}$ were divided into routine clinical medicine plus Nao-Xue-Shu oral liquid $(n=78)$ as treatment group, and routine clinical medicine $(n=80)$ only served as the control group. The incidence of SBI and the classification of a favorable prognosis and a bad prognosis using the Glasgow outcome scale (GOS) were assessed to evaluate the clinical effects. The changes of IL- 6 and TNF- $\alpha$ levels were determined to study the mechanism of the effects for the TCM. Results. The incidence of SBI at the end of week 2 was $8.97 \%$ in the treatment group and $23.75 \%$ in the control group, and the difference was significant $(P<0.001)$. The incidence of a favorable prognosis was $48.72 \%$ in the treatment group and $32.72 \%$ in the control group, and the difference was significant $(P<0.01)$ at the end of week 2 . These findings indicate clear differences for IL- 6 and TNF- $\alpha$ at the end of week 1 and week 2 compared with before treatment for the treatment group and a marked difference at the end of week 2 between the two groups. It also shows a significant difference between the end of week 2 and before treatment for IL- 6 and TNF- $\alpha$ for the control group, although the difference was much smaller than the treatment group. Conclusion. Nao-Xue-Shu oral liquid could protect against the occurrence of SBI and improve HCH and SBI patients. It may also decrease the damage and the mass effects of the hematoma by reducing IL- 6 and TNF- $\alpha$ to obtain the effects, and thus it is a potentially suitable drug for $\mathrm{HCH}$ and SBI.

\section{Introduction}

Secondary brain insults (SBI) predominantly due to hypotension are frequent among patients with fatal traumatic brain injury. SBI following primary impact (e.g., secondary hemorrhage insults) are important causes of damage to the brain [1, 2]. If the SBI is caused by hypertensive cerebral hemorrhage $(\mathrm{HCH})$, it will be defined as hypertensive cerebral hemorrhage SBI (HCH-SBI). In the past two decades, including animal experiments and clinical trials, a great number of studies have been carried out on HCH-SBI. It has been shown that hypotension or hypoxia $[3,4]$, intraoperative hypotension [5], and hyperglycemia in the intensive care unit of HCH-SBI are consistently associated with poor outcomes in $\mathrm{HCH}$ patients. Hypotension is one of the most significant symptoms of SBI after head injuries [6]. The combination of hypotension and head injury is associated with increased mortality and morbidity in comparison with hypertensive cerebral hemorrhage alone [7]. Despite animal studies indicating encouraging results, however, human trials assessing the use of pharmacological agents after SBI have all failed to show efficacy. Proinflammatory factors such as serum interleukin 6 (IL-6) and tumor necrosis factor $\alpha$ (TNF- $\alpha$ ) may indicate potential mechanism of the brain injury [8] and 
may investigate pathogenesis of $\mathrm{HCH}-\mathrm{SBI}$. Nao-Xue-Shu oral liquid [9], a traditional Chinese medicine (TCM), is often used to treat $\mathrm{HCH}$ in China and decrease the incidence of SBI. Therefore, the current study is directed towards providing an optimal physiological environment in order to minimize SBI and maximize the body's own regenerative processes.

\section{Subjects and Methods}

2.1. Subjects. A total of 158 patients with $\mathrm{HCH}$ from our two hospitals (93 cases from Shuguang Hospital and 65 from Shanghai Seventh Hospital) were collected to participate in the study. All of the patients had been diagnosed with hypertension before suffering from a cerebral hemorrhage and 147 cases had a higher blood pressure level than normal when the cerebral hemorrhage occurred. The remaining 11 cases were excluded due to a second cerebral hemorrhage, such as an arterial aneurysm, moyamoya disease, cerebral arteriovenous malformation, cerebral venous sinus thrombosis, and CADASIL (cerebral autosomal dominant arteriopathy with subcortical infarcts and leukoencephalopathy) and were classified as having $\mathrm{HCH}$. The cerebral hemorrhage was the first in 151 cases and the second in 7 cases. Intracerebral hemorrhage of the patients was confirmed by computed tomography (CT). The size of an intracerebral hematoma was calculated using the $A B C / 2$ formula [2], as follows: the volume of an ellipsoid is $4 / 3 \pi(A / 2)(B / 2)(C / 2)$, where $A, B$, and $C$ are the three diameters. If $\pi$ is estimated to be 3 , then the volume of an ellipsoid becomes $A B C / 2$. Patients were divided into the treatment group (treatment plus Nao-XueShu oral liquid, $n=78$ ) and control group (treatment without Nao-Xue-Shu oral liquid, $n=80$ ) in a single-blind fashion. No significant differences in gender, age, number of cases, volume of hematoma, duration, or types of diseases between the two groups were found, and the 2 groups were comparable (Table 1). Inclusion criteria for the patients with SBI were (1) high temperature $\geq 39$ degrees for more than 4 hours; (2) blood pressure $\leq 90 / 60 \mathrm{mmHg}$ for more than 2 hours; (3) oxygen partial pressure $\left(\mathrm{PaO}_{2}\right) \leq 60 \mathrm{mmHg}$; (4) fasting blood glucose $\geq 9 \mathrm{mmol} / \mathrm{L}$; and (5) electrolyte disorder and acidbase imbalance. If the patients suffered symptoms or signs or had more than 3 changes in laboratory values such as blood pressure and $\mathrm{PaO}_{2}$, the patient was defined as suffering from SBI.

2.2. Treatment Methods. The control group underwent routine clinical treatments and measurements according to the guidelines of Western medicine [10], including monitoring electrocardiograph (ECG) and blood pressure fluctuations. To control blood pressure and intracranial pressure, mannitol and/or furosemide and citicoline were administered by intravenous infusion according to the patient's situation and the blood pressure was maintained at less than $180 / 90 \mathrm{mmHg}$. The patients in the treatment group were treated using the same routine treatments as the control group and were also given $10 \mathrm{~mL}$ of $\mathrm{Nao}$-Xue-Shu oral liquid $[9,11]$, three times per day (Shandong Wohua Pharmaceutical Polytron Technologies Inc.), which consists of Astragalus root, Hirudo, Acorus gramineus, radix achyranthis bidentatae, tree peony bark,
Rheum officinale, and Ligusticum wallichii (batch number: 5040507 and 5040711). Patients who could not ingest the liquid orally were given it by nasal feeding. The patients in the treatment group took $\mathrm{Nao}-\mathrm{Xue}$-Shu oral liquid for 2 weeks. The clinical and laboratory parameters were measured before treatment (baseline) and at the end of week 1 and week 2 to evaluate the effects of treatment in the two groups.

2.3. Observation Items and Assessments. The blood pressure, respiration, heartbeat, temperature, and arterial oxygen saturation (SpO2) of most patients were monitored with an electrocardiograph. Blood gas analysis and blood sugar were tested once per day. The Glasgow Outcome Scale (GOS) and the incidence of SBI were the main outcome measures of the study. The serum interleukin 6 (IL-6) and tumor necrosis factor $\alpha$ (TNF- $\alpha$ ) levels were determined 3 times (before treatment, at the first weekend, and at the second weekend) as the secondary outcomes. Liver and kidney function were also tested to monitor the side effects of the Nao-Xue-Shu oral liquid.

The Glasgow Outcome Score is used to assess patients with brain damage and enables the objective assessment of their recovery into 5 categories. The score is used to predict the long-term course of rehabilitation to return to work and everyday life [12]. It has five degrees: the first degree (I) death: severe injury or death without recovery of consciousness; the second degree (II) persistent vegetative state: severe damage with prolonged state of unresponsiveness and a lack of higher mental functions; the third degree (III) severe disability: severe injury with permanent need for help with daily living; the fourth degree (IV) moderate disability: no need for assistance in everyday life and employment possible but may require special equipment; and the fifth degree (V) low disability: light damage with minor neurological and psychological deficits. We defined the prognosis into a poor prognosis (I-III) group and a favorable prognosis (IV-V) group.

2.4. Statistics. SPSS 18.0 software package was used for statistical analysis. Data are presented as the mean and standard deviation $(-x+s)$ or percentage (\%). Chi-square test was used to test the differences of sex and area of the cerebral hemorrhage; $t$-test was conducted to check the differences of age, blood pressure, and volumes of the hematoma of the baseline of the two groups. Repeated-measure ANOVA was conducted to test the differences among changes in outcomes at baseline and at the end of week 1 and week 2 for both groups followed by post hoc Fisher test whenever necessary. Differences of the IL- 6 and TNF- $\alpha$ levels and the volume of the hematoma were obtained by the questionnaire and were assessed using the Friedman test with subsequent post hoc verification using Wilcoxon test. $P<0.05$ was considered to indicate a statistically significant difference.

\section{Results}

No significant differences in age, sex, baseline of IL-6, or baseline of TNF- $\alpha$ were found between the two groups, and 
TABLE 1: General characteristics of the two groups.

\begin{tabular}{lccccc}
\hline & Treatment & Control & Statistic & $P$ value $95 \%$ CI (treatment/control) \\
\hline M/F & $47 / 31$ & $50 / 30$ & $\chi^{2}=0.084$ & 0.772 & - \\
Age $(y)$ & $64.19 \pm 7.26$ & $63.08 \pm 9.81$ & $t=0.807$ & 0.421 & $62.58-65.81 / 60.93-65.23$ \\
Onset time BP (SBP) (mmHg) & $173.37 \pm 29.55$ & $169.89 \pm 30.62$ & $t=0.727$ & 0.469 & $166.81-179.93 / 163.18-176.60$ \\
Onset time BP (DBP) (mmHg) & $109.83 \pm 12.69$ & $110.31 \pm 13.15$ & $t=0.233$ & 0.816 & $107.01-112.65 / 107.42-113.19$ \\
Volume (mL) & $38.64 \pm 14.52$ & $36.91 \pm 19.37$ & $t=0.634$ & 0.527 & $35.42-41.86 / 32.67-41.15$ \\
Outside of putamen (putamen and external capsule) $(n)$ & 37 & 36 & & & \\
Inside of putamen (globus pallidus and internal capsule) $(n)$ & 19 & 21 & & & \\
Subcortex $(n)$ & 9 & 10 & $\chi^{2}=0.560$ & 0.967 \\
Cerebellum $(n)$ & 7 & 5 & & & \\
Thalamus and broken into ventricles $(n)$ & 7 & 8 & & &
\end{tabular}

TABLE 2: Quantitative changes of interleukin 6 (IL-6), tumor necrosis factor $\alpha$ (TNF- $\alpha$ ), and the volume of hematoma before and after the additional treatment in the treatment and control groups.

\begin{tabular}{lcccc}
\hline Group & Time points & IL-6 $(\mathrm{pg} / \mathrm{mL})$ & TNF- $\alpha(\mathrm{pg} / \mathrm{mL})$ & Volume $(\mathrm{mL})$ \\
\hline \multirow{3}{*}{ Treatment $(n=74)$} & Baseline & $84.26 \pm 12.47$ & $186.92 \pm 36.55$ & $38.64 \pm 14.52$ \\
& Week 1 & $53.83 \pm 27.64^{* \#}$ & $121.34 \pm 27.66^{* \#}$ & $29.37 \pm 10.58^{* * \#}$ \\
& Week 2 & $21.08 \pm 15.39^{* * * \# \# \#}$ & $73.59 \pm 36.72^{* * * \# \# \#}$ & $17.81 \pm 9.37^{* * * \# \#}$ \\
\hline \multirow{2}{*}{ Control $(n=72)$} & Baseline & $87.09 \pm 12.47$ & $183.33 \pm 43.35$ & $36.91 \pm 19.37$ \\
& Week 1 & $72.63 \pm 25.58$ & $169.28 \pm 35.91$ & $31.96 \pm 13.86$ \\
& Week 2 & $50.71 \pm 19.07^{*}$ & $108.35 \pm 45.49^{* *}$ & $25.95 \pm 12.36^{* *}$ \\
\hline
\end{tabular}

Note: ${ }^{*} P<0.05,{ }^{* *} P<0.01$, and ${ }^{* * *} P<0.001$ compared with baseline of the same group; ${ }^{\#} P<0.05,{ }^{\# \#} P<0.01$, and ${ }^{\# \#} P<0.001$ compared with control group for the same time points.

there were no significant differences in liver laboratory tests and kidney functions among baseline, week 1, and week 2 between the treatment and control groups (Tables 1 and 2). At the end of week 2, there were 12 deaths: 4 patients in the treatment group (who died on days 5, 7, 10, and 11) and 8 patients in the control group ( 2 died on day 2, 2 on day 5 , 3 on day 7 , and one on day 10$)$.

After two weeks of treatment, 7 patients (8.97\%) in the treatment group and 19 (23.75\%) in the control group had SBI, and the difference between the two groups was significant $(P<0.001$, Ridit analyses). During the weekend of week 2 , the GOS outcome was a favorable prognosis in 38 cases $(48.72 \%)$ in the treatment group but in only 25 cases $(31.25 \%)$ in the control group, and the difference was significant (Ridit analysis, $P<0.01$ ). At the end of the study, no significant differences were found such as temperature, blood pressure, oxygen partial pressure, fasting blood glucose, and electrolyte disorder and acid-base imbalance compared between the two groups.

Compared with baseline, IL- 6 and TNF- $\alpha$ had decreased at the end of week 1 and week 2 in the treatment group $(P<$ 0.05 for both laboratory factors of week 1 and $P<0.001$ for both laboratory factors at the end of week 2), while the factors in the control group only indicated a significantly decreased outcome at the end of week 2 compared with its baseline $(P<0.05$ for IL-6 and $P<0.01$ for TNF$\alpha$ ), and the decreased level at the end of week 2 was much smaller than that in the treatment group $(P<0.001$ for both factors, Table 2). The same differences were also found when comparing the two groups by considering the volume of hematoma of the cerebral hemorrhage. The volume of the hematoma had decreased at the end of week 1 and week 2 in the treatment group, while the volume in the control group only indicated a significantly decreased outcome at the end of week 2 compared with its baseline, and the decreased level at the end of week 2 was much smaller than that in the treatment group (Table 2).

\section{Discussion}

Our results indicated that, compared with the control group, the treatment group (Nao-Xue-Shu oral liquid) had a lower incidence of SBI at the end of week 2. Furthermore, the treatment group exhibited clinical improvements in the patients with $\mathrm{HCH}-\mathrm{SBI}$, indicating that Nao-Xue-Shu oral liquid can be used as an additional treatment for $\mathrm{HCH}$ and $\mathrm{HCH}-\mathrm{SBI}$.

$\mathrm{HCH}$ is a common stroke of cerebral hemorrhage in China, has a higher disability rate and higher mortality rate, and is extremely difficult to prevent and treat by modern medicine. Integrative treatment may have additional effects in treating serious and difficult diseases [13-15] such as the occurrence of SBI of $\mathrm{HCH}$. This study demonstrated that $\mathrm{Nao}-\mathrm{Xue}$-Shu oral liquid could significantly decrease the toxicity of hematoma in the brain by removing IL- 6 and TNF$\alpha$ (Table 2), preventing the occurrence of SBI and improving the recovery of neuronal function, and, finally, decreasing the disability and mortality rates of the patients.

SBI was first defined by Miller et al. who studied the insults due to traumatic brain injury [1]. They suggested 
that the higher disability rate and higher mortality rate of brain injury were not only caused by the traumatic brain injury directly, but also derived from SBI, the mass effect of the hematoma, and peripheral toxicity from the hematoma. Matsushita et al. hypothesized that cell death after intracerebral hemorrhage may be mediated in part by apoptotic mechanisms. They provided initial evidence that apoptotic mechanisms may mediate some of the injury in brain after intracerebral hemorrhage [16]. Xu et al. observed SBI induced by traumatic brain injury, including excitotoxicity, oxidative stress, inflammatory response, and neuronal degeneration, and indicated that mouse brain with traumatic brain injury can be protected by inhibiting the inflammatory response and that inhibiting inflammatory-induced autophagy may play a pivotal role in its neuroprotection [8]. Zhang et al. reported the levels of serum IL- 6 and TNF- $\alpha$ were increased significantly during the early stage of $\mathrm{HCH}$ [17]. Suzuki et al. investigated the pathogenesis of hypertensive cerebrovascular lesions by immunohistochemistry and scanning electron microscopy. The brains of rats with experimentally induced hypertension exhibited severe edema and intracerebral hemorrhage. They found that IL-6, IL-8, and TNF- $\alpha$ endothelial cell expression was upregulated and suggested that hypertension activates endothelial cells to increase the expression of adhesion molecules and cytokines and induces neutrophil and monocyte adhesion and migration, resulting in endothelial cell injury and increased permeability of endothelial cells, which results in hypertensive arterial disease [18].

$\mathrm{HCH}-\mathrm{SBI}$ is an acute onset and rapid progression vascular disease, and it has a serious impact on the quality of life and safety of the patient because it can result in higher level brain function disturbances such as coma, aphasia, dementia, and epilepsy. Explaining the mechanism of action of $\mathrm{Nao}-\mathrm{Xue}-\mathrm{Shu}$ oral liquid in TCM theory may be difficult to understand for most Western doctors [9]. HCH-SBI in TCM is explained as "apoplexia" and an "attack on the viscera and bowels" [19], caused by a $Q i$ deficiency, blood stasis, and phlegm. Due to the $Q i$ deficiency, the blood stasis and phlegm obstruct the internal structure of blood vessels that then intertwist with each other, and the abnormal blood causes intervessel high blood pressure, and forcing the static blood with phlegm out of the blood vessel may break the vessel, leading to hemorrhage [20,21]. The blood stasis and phlegm may be expressed as "inflammatory" and "abnormal blood viscosity" in Western medicine [9], In TCM theory, if blood stasis is accompanied by phlegm, it can lead to a more significantly damaged lesion in the brain [19]. This is the mechanism that explains why $\mathrm{HCH}$ patients often also have advanced neuronal damage, including coma, aphasia, and epilepsy. Physiologically, cleaning and powerful Qi (Qing-yang Qi) can supply energy to the brain to maintain its function and collect and modulate the blood and force it to circulate in the correct way in brain blood vessels $[9,19,20]$. If the circulation has been obstructed by the blood stasis with phlegm, the occlusion of blood vessel orifices will occur and the power of $Q i$ will decrease; Qing-yang $Q i$ is also like nutrition for the brain; if it cannot rise, it can lead to the brain lacking sufficient energy to maintain awaking and thinking and can even cause lethargy and coma. If the Qing-yang Qi deteriorates further, blood pressure may not be maintained, hypotension will occur, and then SBI may develop. When treating this disease, we should consider three TCM pathogenic matters: Qi, blood stasis, and phlegm. First, we should eliminate Qingyang $\mathrm{Q} i$, which can modulate blood circulation and control or decrease bleeding. Astragalus root as a major component in Nao-Xue-Shu oral liquid can provide a stronger Qingyang $\mathrm{Q} i$ [9]. $\mathrm{Q} i$ also provides energy to raise the nutrient level in the blood to the brain when treating the ischemia and improves the level of consciousness [20]. In TCM, Qi can improve circulation throughout the entire system and enhances metabolism. The other main component in the oral liquid is Hirudo, a type of earthworm that has been used for more than one thousand years in China, which can rapidly eliminate blood stasis and treat the second pathogenic condition, that of blood stasis [21,22], without any side effects such as bleeding. Other than these two components, the $\mathrm{Nao}-\mathrm{Xue}-\mathrm{Shu}$ oral liquid formulation contains 5 other TCM herbs that can help increase $Q i$, remove blood stasis and phlegm, assist the body to excrete the pathogenic metabolites of blood stasis and phlegm, and finally decrease the IL-4, IL-6, IL8, and TNF- $\alpha$ levels [23]. Several studies [24-27] have provided strong evidence that TCM, which promote blood circulation to remove blood stasis functions, could diminish inflammation by decreasing IL-6, IL-8, and TNF- $\alpha$ factors. In fact, Nao-Xue-Shu oral liquid contains two famous prescriptions of TCM. One is Bu-Yang-Huan-Wu decoction, which originated in the Qing Dynasty (about 185 years ago in 1830 ) and has been used frequently to treat stroke in China and Asia [27]. The other is Da-Huang-Shu-Chong pill, which comes from the very famous TCM text Iin-Gui-Yao-Lue (By Zhang Zhongjing, about 1700 years ago) and has been used to remove blood stasis from the body [28, 29]. The combination of these 2 prescriptions is the most effective treatment for treating $\mathrm{HCH}$ with SBI. Clinical pharmacological studies have confirmed that Nao-Xue-Shu oral liquid accelerates the absorption of hematoma in the brain of rats, reduces edema around the hematoma accelerating fibrinolysis and inhibiting thrombosis, increases cerebral blood flow, and improves brain blood and oxygen supply, thereby improving blood circulation and promoting the absorption of hematoma [30].

In this study in patients with $\mathrm{HCH}$, compared with the control group, patients in the treatment group had a markedly lower incidence of SBI. Nao-Xue-Shu oral liquid may decrease the mass effects of hematoma, improve the absorbance of hemorrhage, eliminate the toxic stimulation of peripheral brain tissue, and inhibit the accumulation of inflammatory factors. Thus, it can prevent the occurrence of SBI as well as treat $\mathrm{HCH}-\mathrm{SBI}$. We did not observe any more side effects based on the results of the laboratory tests.

Chinese medicine has the distinctive function of modulating the body or dealing with diseases, including treating brain problems [31, 32]. We are still unable to show how the ingredients pass through the blood-brain barrier (BBB), but they have been used in many countries for treating many diseases [14]. Nao-Xue-Shu oral liquid contains a type of worm and this may be one problem in terms of ethics or acceptability as it may be difficult to introduce such a treatment into some foreign countries, although worms are 
frequently used in TCM treatments and TCM researchers in China have demonstrated that they are harmless and safe. Non-RCT and insufficient cases are the other shortages of our study. In order to validate the causes of the disease based on clinical data, large-scale, multicenter, double-blind randomized control studies may be needed to verify the effectiveness of $\mathrm{Nao}-\mathrm{Xue}-\mathrm{Shu}$ oral liquid in the treatment of $\mathrm{HCH}$ and $\mathrm{HCH}-\mathrm{SBI}$.

\section{Disclosure}

Hongning Jiang and Ying Qin contributed as co-first authors.

\section{Competing Interests}

The authors declare that they have no competing interests.

\section{Acknowledgments}

This study was sponsored and supported by the National Natural Science Foundation of China (81373619).

\section{References}

[1] J. D. Miller, R. C. Sweet, R. Narayan, and D. P. Becker, "Early insults to the injured brain," The Journal of the American Medical Association, vol. 240, no. 5, pp. 439-442, 1978.

[2] R. U. Kothari, T. Brott, J. P. Broderick et al., "The ABCs of measuring intracerebral hemorrhage volumes," Stroke, vol. 27, no. 8, pp. 1304-1305, 1996.

[3] Y. Tian, S.-X. Guo, J.-R. Li et al., "Topiramate attenuates early brain injury following subarachnoid haemorrhage in rats via duplex protection against inflammation and neuronal cell death," Brain Research, vol. 1622, pp. 174-185, 2015.

[4] A. I. Maas, H. F. Lingsma, and B. Roozenbeek, "Predicting outcome after traumatic brain injury," in Handbook of Clinical Neurology, vol. 128, pp. 455-474, 2015.

[5] J. D. Miller, I. R. Piper, and P. A. Jones, "Integrated multimodality monitoring in the neurosurgical intensive care unit," Neurosurgery clinics of North America, vol. 5, no. 4, pp. 661-670, 1994.

[6] K. Matsushita, W. Meng, X. Wang et al., "Evidence for apoptosis after intercerebral hemorrhage in rat striatum," Journal of Cerebral Blood Flow and Metabolism, vol. 20, no. 2, pp. 396-404, 2000.

[7] Z. Du, J. Liu, H. Jia, W. Xu, and X. Zhao, “Three hydrogenrich solutions protect against intestinal injury in uncontrolled hemorrhagic shock," International Journal of Clinical and Experimental Medicine, vol. 8, no. 5, pp. 7620-7626, 2015.

[8] J. Xu, H. Wang, X. Lu et al., "Posttraumatic administration of luteolin protects mice from traumatic brain injury: implication of autophagy and inflammation," Brain Research, vol. 1582, pp. 237-246, 2014.

[9] Y. Yan, M. Wang, L. Zhang et al., "Nao-Xue-Shu oral liquid improves aphasia of mixed stroke," Evidence-Based Complementary and Alternative Medicine, vol. 2015, Article ID 709568, 6 pages, 2015.

[10] Y.-N. Huang, "Standardized diagnosis and management in reducing fatality rate of patients hospitalized with acute stroke," Chinese Journal of Neurology, vol. 38, no. 1, pp. 17-21, 2005.
[11] M. Wang, L. Zhang, W. Jiang, W. Pan, and H.-N. Jiang, "Clinical observation of nourishing qi, resolving stagnation, cooling blood and eliminating phlegm method in treating aphasia of mixed stroke," Shanghai Zhong Yi Yao Da Xue Xue Bao, vol. 29, no. 3, pp. 23-30, 2015.

[12] B. Jennett and M. Bond, "Assessment of outcome after severe brain damage," The Lancet, vol. 305, no. 7905, pp. 480-484, 1975.

[13] W. Pan, X. Chen, J. Bao et al., "The use of integrative therapies in patients with amyotrophic lateral sclerosis in Shanghai, China," Evidence-Based Complementary and Alternative Medicine, vol. 2013, Article ID 613596, 6 pages, 2013.

[14] W. Pan and H. Zhou, "Inclusion of integrative medicine in clinical practice," Integrative Medicine International, vol. 1, no. 1, pp. 1-4, 2014.

[15] X. Chen and W. Pan, "The treatment strategies for neurodegenerative diseases by integrative medicine," Integrative Medicine International, vol. 1, no. 4, pp. 223-225, 2015.

[16] K. Matsushita, W. Meng, X. Wang et al., "Evidence for apoptosis after intracerebral hemorrhage in rat striatum," Journal of Cerebral Blood Flow and Metabolism, vol. 20, no. 2, pp. 396-404, 2000.

[17] X.-M. Zhang, X.-L. Li, S.-H. Tang, and Q.-C. Liu, "Effect of head hypothermia on serum inflammatory cytokines levels in patients hypertensive intracerebral hemorrhage," Chinese Critical Care Medicine, vol. 18, no. 5, pp. 294-296, 2006.

[18] K. Suzuki, N. Masawa, and M. Takatama, "The pathogenesis of cerebrovascular lesions in hypertensive rats," Medical Electron Microscopy, vol. 34, no. 4, pp. 230-239, 2001.

[19] D.-H. Yi, Y. Li, S.-X. Shao, Y.-M. Xie, and Y. Yuwen, "Evaluation of the conjoint efficacy in Chinese medicine with the longitudinal latent variable linear mixed model," Chinese Journal of Integrative Medicine, vol. 19, no. 8, pp. 629-635, 2013.

[20] J. Zhu, "Textual research and explanation of 'Qi-Huang," Zhonghua yi shi za zhi, vol. 32, no. 4, pp. 200-204, 2002.

[21] L. Gong, J. Wang, L. Kong, L. Xu, F. Qiu, and Z. Fei, "Clinical observation on removing-stasis, resolving-phlegm and unblocking-fu method in treating patients with hypertensive cerebral hemorrhage after operation," Shanghai Zhong Yi Yao Da Xue Xue Bao, vol. 29, no. 4, pp. 24-26, 2015.

[22] S. Lemke, C. Müller, E. Lipke, G. Uhl, and J.-P. Hildebrandt, "May salivary gland secretory proteins from hematophagous leeches (Hirudo verbana) reach pharmacologically relevant concentrations in the vertebrate host?" PLOS ONE, vol. 8, no. 9, Article ID e73809, 2013.

[23] D. Xie, B. Xu, Y. Sun et al., "Nao-Xue-Shu liquid for hemorrhagic stroke," Zhong Xi Yi Jie He Xin Nao Xue Guan Bing Za Zhi, vol. 5, no. 8, pp. 690-691, 2007.

[24] Y.-Q. Yang and T. Xue, "Clinical study on Nao-Xue-Shu Oral Liquid combined with nimodipine in treatment of hypertensive cerebral hemorrhage," Xian Dai Yao Wu Yu Lin Chuang, vol. 30, no. 11, pp. 1341-1344, 2015.

[25] L.-H. Shaw, L.-C. Lin, and T.-H. Tsai, "HPLC-MS/MS analysis of a traditional Chinese medical formulation of Bu-Yang-HuanWu-Tang and its pharmacokinetics after oral administration to rats," PLoS ONE, vol. 7, no. 8, Article ID e43848, 2012.

[26] C. Wen, H. Xu, and Q.-F. Huang, "Effect of drugs for promoting blood circulation on blood lipids and inflammatory reaction of atherosclerotic plaques in ApoE gene deficiency mice," Zhongguo Zhong Xi Yi Jie He Za Zhi, vol. 25, no. 4, pp. 345-349, 2005. 
[27] L. Cao, B. Han, T. Li, and P. Ma, “The empirical study of IL-6 with promoting blood flow to removing stasis and soften hard lumps and dispel nodes to treat endometriosis," Henan Zhong Yi Xue Yuan Xue Bao, vol. 22, no. 6, pp. 133-134, 2007.

[28] Z. Lai, S. Y. Wang, X. Y. Geng, C. Q. Deng, and R. Z. Zhang, "Effects of Bu Yang Huan Wu decoction on astrocytes after cerebral ischemia and reperfusion," Zhongguo Zhong Yao Za Zhi, vol. 27, no. 10, pp. 763-765, 2002.

[29] T. Jiang and H. Fu, "Progress of experimental studies on prescriptions designed by Zhang Zhongjing," Journal of Traditional Chinese Medicine, vol. 16, no. 1, pp. 55-64, 1996.

[30] X. Ai and C. Liu, "The study of cleaning volume of hematoma and protecting neuron function of hemorrhagic rat model by Nao-Xue-Shu liquid," Zhong Guo Zhong Xi Yi Jie He Xin Nao Xue Guan Bing Za Zhi, vol. 11, no. 7, pp. 859-861, 2014.

[31] W. Pan, Q. Wang, S. Kwak et al., "Shen-Zhi-Ling oral liquid improves behavioral and psychological symptoms of dementia in Alzheimer's disease," Evidence-Based Complementary and Alternative Medicine, vol. 2014, Article ID 913687, 6 pages, 2014.

[32] J. Shen, X. Chen, X. Chen, and R. Deng, "Targeting neurogenesis: a promising therapeutic strategy for post-stroke treatment with Chinese herbal medicine," Integrative Medicine International, vol. 1, no. 1, pp. 5-18, 2014. 


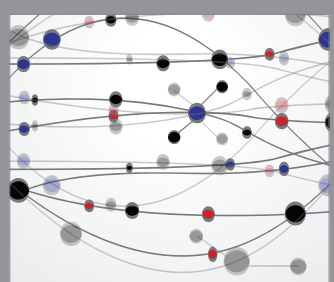

The Scientific World Journal
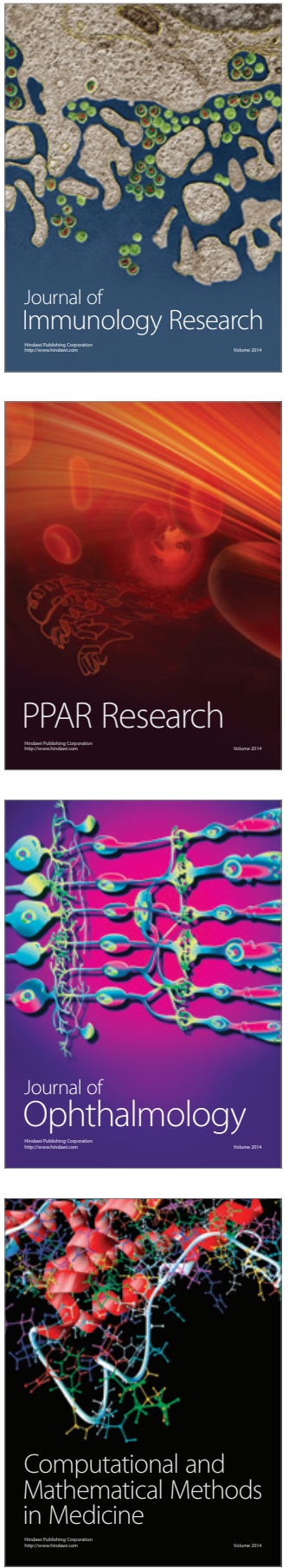

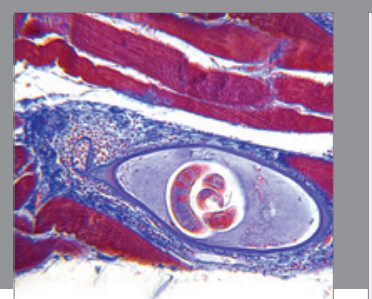

Gastroenterology Research and Practice

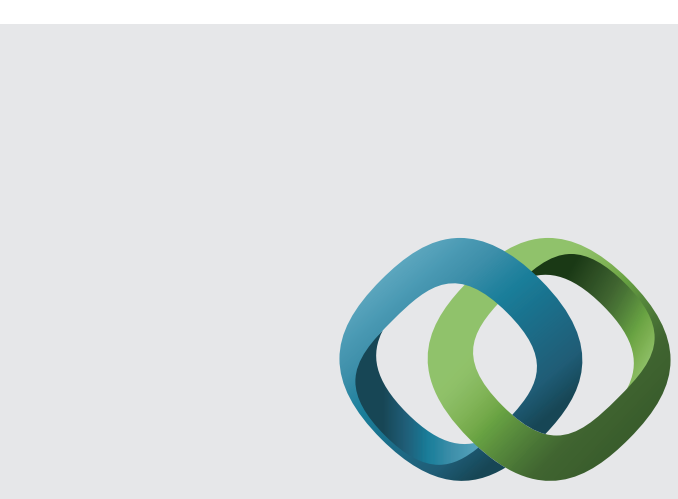

\section{Hindawi}

Submit your manuscripts at

http://www.hindawi.com
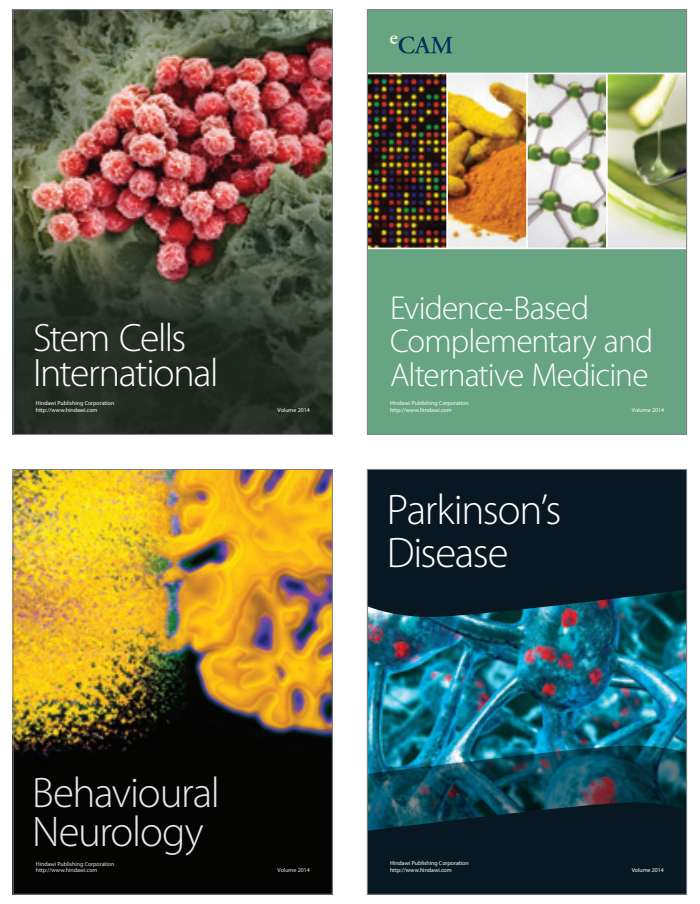
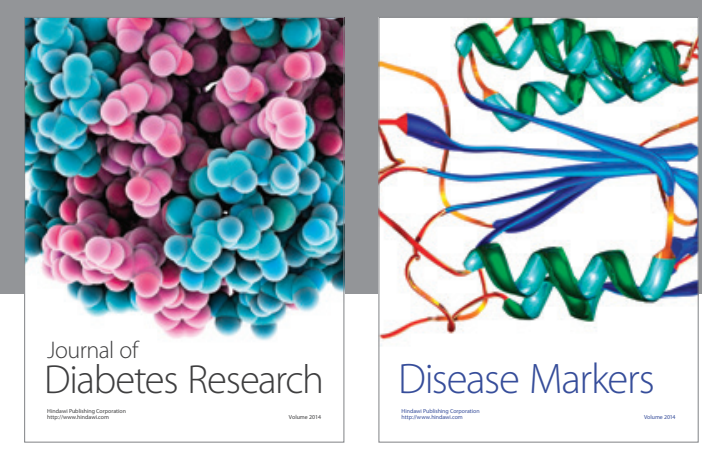

Disease Markers
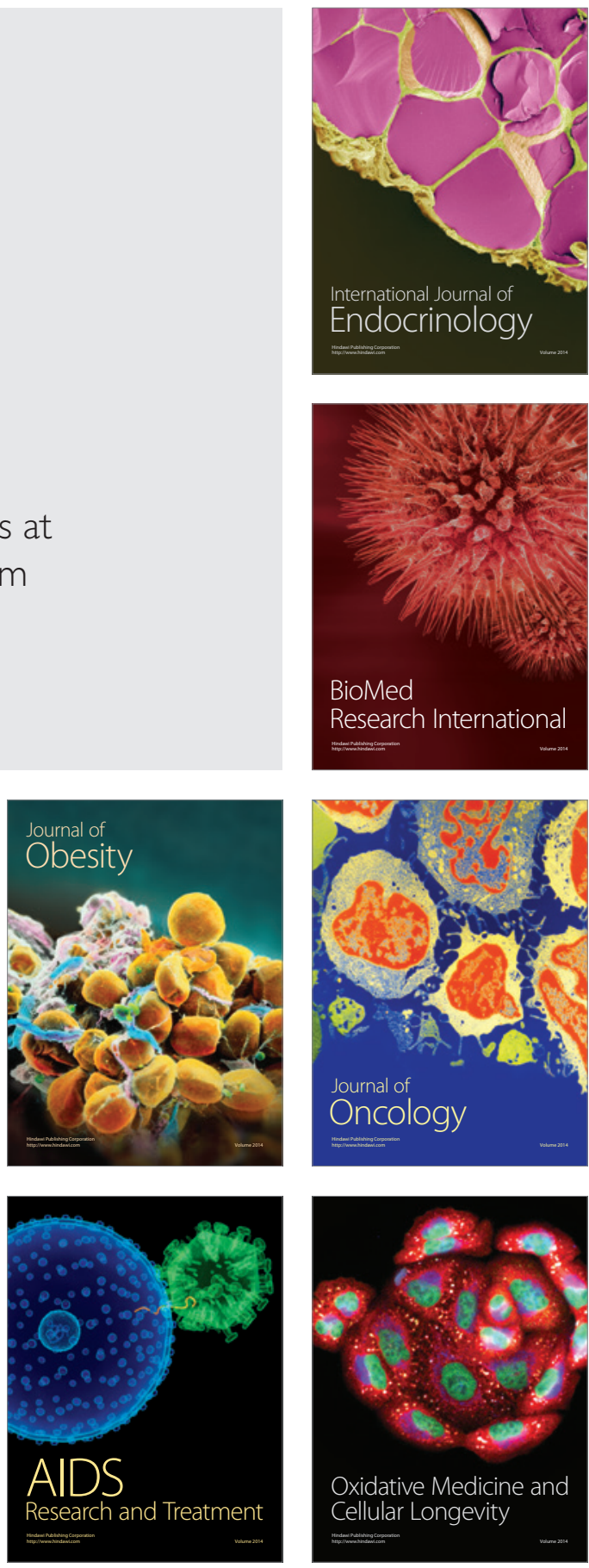